\title{
Generic scenarios of the accelerating Universe
}

\author{
Marek Szydłowski
}

Received: 30 December 2011 / Accepted: 24 January 2012 / Published online: 1 February 2012

(C) The Author(s) 2012. This article is published with open access at Springerlink.com

\begin{abstract}
Recent astronomical observations strongly indicate that the current Universe is undergoing an accelerated phase of its expansion. If the Universe evolution is described by the FRW model then the acceleration should be driven by some perfect substance violating the strong energy condition. Hence the negative pressure is required for the explanation of acceleration. While different candidates for the fluid termed dark energy have been suggested, the positive cosmological constant seems to be the simplest candidate for dark energy description. However the Lambda term treated as a quantum vacuum energy has no simple physical interpretation because of the fine tuning problem. In this paper we characterize a certain class of evolutional scenario of the accelerating universe from the point of view genericity expressed in notion of structural stability. It is shown that in this class all evolutional scenarios with transient acceleration effect are not generic. Only the LCDM model with the acceleration phase following the deceleration phase is structurally stable. Because the cosmological models with transient acceleration appear in string theory we conclude that they are not generic.
\end{abstract}

Keywords Cosmology $\cdot$ Acceleration $\cdot$ Structural stability

\footnotetext{
M. Szydłowski ( $\varangle)$

Astronomical Observatory, Jagiellonian University, Orla 171, 30-244 Kraków, Poland

e-mail: marek.szydlowski@uj.edu.pl

Present address:

M. Szydłowski

Mark Kac Complex Systems Research Centre, Jagiellonian

University, Reymonta 4, 30-059 Kraków, Poland
}

\section{Introduction}

Recent astronomical observations of distant supernovas SNIa type strongly indicate that the current Universe is undergoing an accelerated phase of expansion (Riess et al. 1995, 1998, 2004; Perlmutter et al. 1998, 1999; Astier et al. 2006). If the Universe evolution is described by homogeneous and isotropic models filled with a perfect fluid then the acceleration should be driven by a perfect fluid violating the strong energy condition. If different candidates for a fluid termed dark energy are suggested, the simple candidates for the dark energy in the form of the positive cosmological constant seems to be the best one (Spergel et al. 2003; Tegmark et al. 2004). While the LCDM model is a good phenomenological description of the acceleration phase of the expansion of the Universe there is a serious problem with the interpretation of the Lambda term as a quantum vacuum energy because of the fine tuning problem (Freese and Lewis 2002; Godlowski et al. 2004). Our studies show that when the LCDM model has the status of an effective theory which offers description of the observational facts rather than their explanation this theory introduces principally the new theoretical element which plays the role of an effective parameter changing dramatically the dynamics.

It has been recently found that varying the equation of state of dark energy allows to obtain the coasting model with diminishing rate of acceleration (Shafieloo et al. 2009). This model which reaches the constant velocity of expansion fits the data as well as the LCDM model. Moreover acceleration as a transient phenomenon appears naturally in quintessence cosmology (Alcaniz 2009). Alcaniz argued that the equation of state parameter switches from freezing to thawing regimes which is manifested by the transient acceleration effect. It is interesting that string theory predicts the possibility of transient cosmic acceleration (Frieman et al. 1995; 
Carvalho et al. 2006). Many examples of the transient cosmic acceleration can be also found in brane world cosmologies (Sahni and Shtanov 2003).

In this paper we characterize a certain class of evolutional scenario of the accelerating universe from the point of view genericity expressed in notion of structural stability. It is shown that in this class all evolutional scenarios with transient acceleration effect are not generic. Only the LCDM model with the acceleration phase following the deceleration phase is structurally stable. Because the cosmological models with transient acceleration appear in string theory we conclude that they are not generic.

\section{Structural stability issues}

Einstein field equations constitute in general very complicated system of partial nonlinear differential equations but in the cosmology important role plays its solutions with some symmetry assumptions postulated at the very beginning. Usually an isotropy, homogeneity itself and its symmetry are assumed. In this case the Einstein field equations can be reduced to the system of ordinary differential equations, i.e. dynamical systems. Hence to the cosmology could be applied the dynamical system methods in natural way. The application of these methods allows to reveal some stability properties of particular visualized in a geometrical way as the trajectories in the phase space. Hence one can see how large is the class of solutions leading to the desired property in tools of the attractors and the inset of limit set (an attractor is a limit set with an open inset-all the initial conditions that end up in the some equilibrium state). The attractors are the most prominent experimentally. It is because the probability of an initial state of the experiment to evolve asymptotically to the limit set it is proportional to the volume of inset.

The idea, now called structural stability emerged early in the history of dynamics investigation in 1930's the writings of Andronov, Leontovich and Pontryagin in Russia (1934) (Andronov and Pontryagin 1937) (the authors do not use the name structural stability but rather the name "rough systems"). This idea is based on an observation an actual state of the system can never be specified exactly and application of the dynamical systems might be useful anyway if it can describe the features of the phase portrait that persist when the state of the system is allowed to move around (see Deffayet et al. 2002 for more comments).

Among all dynamicists there is shared prejudices that:

1. There is a class of phase portraits that are far simpler than arbitrary ones which can explain why a considerable portion of the mathematical physics has been dominated by the search for the generic properties. The exceptional case should not arise very often in application and they de facto interrupt discussion (classification) (Deffayet et al. 2002, p. 349).

2 . The physically realistic models of the world should possess some kind of the structural stability because having so many dramatically different models all agreeing with observation would be fatal for the empirical method of science (Shtanov 2000; Shtanov and Sahni 2003, see also Thom 1977; Szydlowski et al. 1984; Biesiada 2003; Golda et al. 1987; Tavakol and Ellis 1988).

These prejudices in the Holton terminology can be treated as a thematic principles (Losee 1993; Holton 1981). In the cosmology a property (for example acceleration) is believed to be "physically realistic" if it can be attributed by the generic subsets of the models within a space of all admissible solutions or if it possesses a certain stability, i.e. if it is shared by a "epsilon perturbed model". For example G. F. R. Ellis (Ellis 1983) formulated so called a probability principle "The Universe model should be one that is a probable model within the set of all universe models" and a stability assumption which states that "the Universe should be stable to the perturbations". The problems are how to define:

1. the space of state and its equivalence,

2. the perturbation of the system.

The dynamical system is called structurally stable if all $\delta$ perturbation of it (sufficiently small) have the epsilon equivalent phase portrait. Therefore for the conception of structural stability we considered a $\delta$-perturbation of vector field determined by the right-hand sides of the system which is small (measured by delta). We also need a conception of the epsilon equivalence. This has the form of topological equivalence-a homeomorphism of the state space preserving the arrow of time on each trajectory. In the definition of structural stability considers only the deformation of "rubber sheet" type stretches or slides the phase space a small amount measured by epsilon.

There are developed other concepts of stability used by some authors (Lidsey 1993; Aguirregabiria and Lazkoz 2004). For example concepts of rigidity and fragility is used in the sense that the attractor solutions never change as long as some conditions are met. In the structural stability conception the global dynamics is important rather than the fragility of solutions against changes in the shape of a functional form of the Hubble function. It is also used the concept of rigidity in the context of a final theory of physics (TOE). Roughly speaking a mathematical structure is said to be rigid, with respect to a certain deformation parameter, if its all deformation with respect to this parameter yields again the same structure (Gerstenhaber 1964; Roger 1991). It is interesting that while the deformation parameter is not defined uniquely, the deformation procedure can be strictly defined. The main advantage of the structural 
Fig. 1 The schematic illustration of (a) structural stability of dynamical system under perturbation;

(b) structural instability of a center; (c) structural stability of a saddle
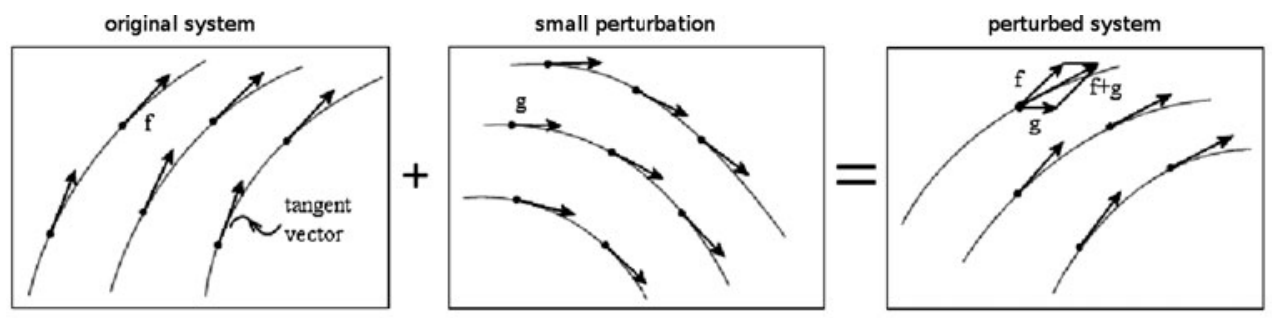

original system - centre
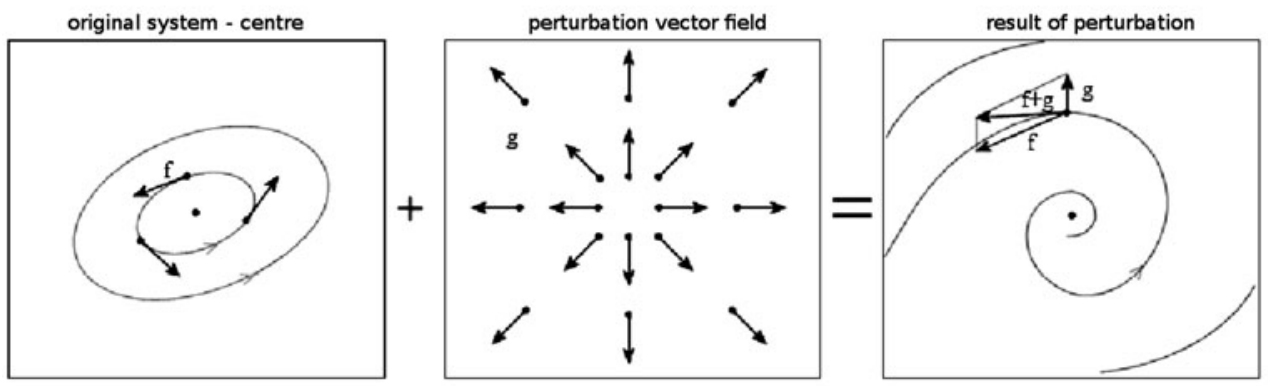

original system - saddle
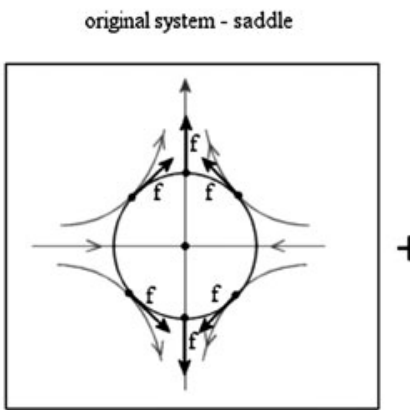

central perturbation
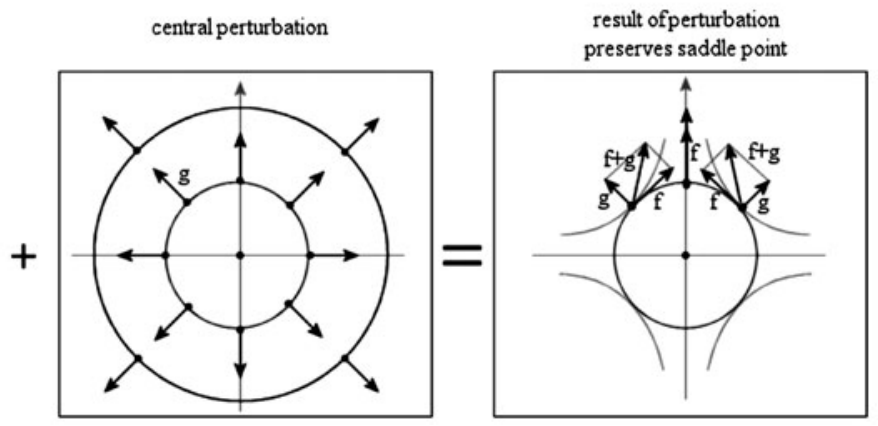

stability is that it is the characterization of global dynamics itself.

Recently, properties of structural stability of cosmological models were investigated by Kokarev (2009) (see also Szydlowski and Czaja 2004a, 2004b). In the introduction to the paper author claimed that the history of cosmology shows that corrections of cosmological models are realized mainly by the sequence of their, in some sense "small", modifications and some of them may "survive" after small changes, while the other may disappear. In the former case the property is referred to as "rough" or structurally stable, in the later one- "thin" or structurally unstable. The author studied how some model properties, like singularities for example, will be present in the model if we "perturb" the model (for example generalize the Lagrangian of General Relativity). In our approach the property of structural stability is the property of the model itself. Also the type of perturbations is not specified (epsilon perturbation idea). Therefore, if we prove the structural instability of CDM model, the result will not depend on the choice of the type of perturbation. Then the property of structural stability becomes its constitutive property at the very beginning without restriction to the class of perturbation induced by considering new theories with generalized Lagrangian.
The problem of structural stability seems to be also important in the context of choosing the initial conditions in cosmology. If the model is structurally stable the given and close to it models characterize the same qualitative dynamics.

Figure 1 illustrates the property of structural stability of single spiral attractor (focus) and saddle point and structural instability of center. The addition of a delta perturbation pointing outward (no matter how weak) results in a point repeller. We call such a system structurally unstable because the phase portrait of the center and focus are not topologically equivalent (notice that all phase curves around the center are closed in contrast to the focus. Hence one can claim that a pendulum system (without friction) is structurally unstable.

Idea of structural stability attempts to define the notion of stability of differential deterministic models of the physical processes. In the case of planar dynamical systems (as in the case of models under consideration) there is true Peixoto theorem (Peixoto 1962) which states that structurally stable dynamical systems form open and dense subsets in the space of all dynamical systems defined on the compact manifold. This theorem is basic characterization of structurally stable dynamical systems in the plane which offers the possibility 
of exact definition generic (typical) and non-generic (exceptional) cases (properties) in tools of the notion of structural stability. Unfortunately there is no counterparts of this theorem in more dimensional case when structurally unstable systems can also form open and dense subsets. For our aims, it is important that Peixoto theorem can give the characterization of generic cosmological models in terms of potential function $V$ of the scale factor $a$ which determine the motion of the system of Newtonian type: $\ddot{a}=-\frac{\partial V}{\partial a}$.

Therefore we can treat FRW equation with various forms of dark energy as the two-dynamical systems which looks like Newtonian type where the role of coordinate variable is played by the cosmological radius (or redshift $z: 1+z=$ $\frac{a_{0}}{a} \equiv x^{-1}$ ). We can construct an effective potential, the second order acceleration equation has exactly the Newtonian form, where the role of a coordinate variable is played by the cosmological radius.

Using the term of the structural stability introduced first by Andronov, Leontovich and Pontryagin in thirties, one can classify different models of cosmic acceleration. It will be demonstrated that models with the accelerating phase which follows the deceleration are natural and typical from the point of view of the dynamical systems theory combined with the notion of structural stability in contrast to the models with bounces. In Fig. 2 there are illustrated two cases: (a) inverted single-well potential and (b) more complicated form of the potential with two maxima corresponding to the saddle point and minimum corresponding to the center (structurally unstable).

Let us introduce the following definition:

Definition 1 If the set of all vector fields $f \in C^{r}(\mathcal{M})(r \geq$ 1) having a certain property contains an open dense subset of $C^{r}(\mathcal{M})$, then the property is called generic.

From the physical point of view it is interesting to know whether certain subset $v$ of $C^{r}(\mathcal{M})$ (representing a class of cosmological accelerating models in our case) contains a dense subset because it means that this property (acceleration) is typical in $V$ (see Fig. 1).

It is not difficult to establish some simple relation between the geometry of potential function and the localization of critical points and its character for the case of dynamical systems of Newtonian type:

1. The critical point of the system under consideration $\dot{x}=$ $y, y=-\frac{\partial V}{\partial x}$ lies always on $x$-axis, i.e. they are representing static universe $y_{0}=0, x=x_{0}$.

2. The point $\left(x_{0}, 0\right)$ is a critical point of the Newtonian system if it is a critical point of the potential function $V(x)$, i.e. $V(x)=E$ ( $E$ is total energy of the system $E=\frac{y^{2}}{2}+V(x) ; E=0$ for the case flat models and $E=\frac{-k}{2}$ in general).
3. If $\left(x_{0}, 0\right)$ is a strict local maximum of $V(x)$, it is a saddle type critical point.

4. If $\left(x_{0}, 0\right)$ is a strict local minimum of the analytic function $V(x)$, it is a center.

5. If $\left(x_{0}, 0\right)$ is a horizontal inflection point of the $V(x)$, it is a cusp.

Therefore the geometry of potential function will determine the critical points as well as its stability. The integral of energy defines the algebraic curves in the phase space $(x, y)$ which are representing the evolution of the system with time. In any case the eigenvalues of the linearization matrix satisfy the characteristic equation $\lambda^{2}+\left.\frac{\partial^{2} V}{\partial x^{2}}\right|_{x=x_{0}}=0$.

\section{Cosmological models as dynamical systems}

The cosmology is based on the Einstein field equation which represents a very complicated system of partial nonlinear differential equation. Fortunately, the majority of main class of cosmological models from the point of view of observational data, belong to the class of the spatially homogeneous ones, for which has sense the absolute cosmological time. As a consequence, the evolution of such models can be reduced to the systems of ordinary differential equations. Hence to the cosmology could be naturally applied the methods of dynamical system theory or qualitative theory of differential equation. Among these class of models especially interesting are the cosmological models with maximally symmetric space sections, i.e. homogeneous and isotropic. They are called Friedmann-Robertson-Walker (FRW) models if source of the gravity is a perfect fluid described in terms of energy density $\varrho$ and pressure $p$, both are the functions of the cosmological time $t$. The FRW dynamics is described by two basic equations

$\ddot{a}=-\frac{1}{6}(\varrho+3 p) a=-\frac{\partial V}{\partial a}$,

$\dot{\varrho}=-3 H(\varrho+p)$,

where the potential $V=-\frac{1}{6} \varrho a^{2}, a$ is the scale factor and $H=d \ln a / d t$ is Hubble's function, a overdot means the differentiation with respect to the cosmological time $t$.

The first equation is a consequence of the Einstein equations for the component $(1,1),(2,2),(3,3)$ and the energy momentum tensor $T_{v}^{\mu}=\operatorname{diag}|-\varrho, p, p, p|$. This equation is called the Raychaudhuri or acceleration equation. The second equation represents the conservation condition $T_{v ; \mu}^{\mu}=0$. It is very strange and unreasonable that such two simple equations satisfactorily describe the Universe evolution at the large scales. Of course there is a more general class of cosmological models called the Bianchi models which has only the symmetry of homogeneity but they do 
not describe the current Universe which is isotropic as indicated measurement of the cosmic microwave background (CMB)radiation.

The system of equations (1) and (2) admit the first integral called the Friedmann equation

$\varrho-3 H^{2}=3 \frac{k}{a^{2}}$,

where $k$ is curvature constant $(0, \pm 1)$ and $\varrho$ plays the role of effective energy density.

If we consider the LCDM model then

$\varrho_{\text {eff }}=\varrho_{\mathrm{m}, 0} a^{-3}+\Lambda$,

i.e. energy density is a sum of (cold) dust matter and dark energy. Therefore the potential function for the flat FRW model assumes the following form

$V=-\frac{\varrho_{\text {eff }} a^{2}}{6}=-\left(\varrho_{\mathrm{m}, 0} a^{-1}+\Lambda a^{2}\right)$

or in terms of redshift

$V(z)=-\frac{1}{6}\left[\varrho_{\mathrm{m}, 0}(1+z)+\Lambda(1+z)^{-1}\right]$

Formally the curvature effects as well as the cosmological constant term can be incorporated into the effective energy density $\left(\varrho_{k}=-\frac{k}{a^{2}} ; \varrho_{\Lambda}=\Lambda ; p_{\Lambda}=-\Lambda\right)$.

To represent the evolutional paths of cosmological models in this form is popular since Peebles' monograph (Peebles 1993) see also (Padmanabhan 1993) and modern applications (Szydlowski and Czaja 2004a, 2004c, Szydlowski 2007 and references therein).

The form of (1) suggests the possible interpretation for the evolutional paths of cosmological models as a motion of a fictitious particle of unit mass in a one-dimensional potential parameterized by the scale factor. Following this interpretation the Universe is accelerating in the domain of configuration space $\{a: a \geq 0\}$ in which the potential is a decreasing function of the scale factor. In the opposite case if potential is a growing function of $a$ the Universe is decelerating. The limit case of zero acceleration corresponds to an extremum of the potential function.

It is useful to represent evolution of the systems in terms of the dimensionless density parameter $\Omega_{i} \equiv \frac{\varrho_{i}}{3 H_{0}^{2}}$, where $H_{0}$ is present value of Hubble's function. For this aim it is sufficient to introduce the dimensionless scale factor $x \equiv \frac{a}{a_{0}}$ which measures the value of $a$ in the units of the present value $a_{0}$ (which we choose) and reparameterize the cosmological time following rule $t \longmapsto \tau: d t\left|H_{0}\right|=d \tau$. Hence we obtain a 2-dimensional dynamical system describing the evolution of cosmological models

$$
\begin{aligned}
& \frac{d x}{d \tau}=y \\
& \frac{d y}{d \tau}=-\frac{\partial V}{\partial x}
\end{aligned}
$$

and

$\frac{y^{2}}{2}+V(x)=\frac{1}{2} \Omega_{k, 0}, \quad 1+z=x^{-1}$,

where $z$ is redshift and

$V(x)=-\frac{1}{2}\left(\Omega_{\mathrm{eff}} x^{2}+\Omega_{k, 0}+\Omega_{\mathrm{Card}, 0} x^{m+2}\right) ;$

with

$\Omega_{\mathrm{eff}}=\Omega_{\mathrm{m}, 0} x^{-3}+\Omega_{x, 0} x^{-3\left(1+w_{x}\right)}$

for dust matter and quintessence matter satisfying the equation of state $p_{x}=w_{x} \varrho_{x}, w_{x}=$ const.

Above the particle-like description of dynamics covers a broad class of models with dark energy for which effective energy density can be parameterized through the scale factor. In particular the above potential $V(x)$ is a part of the very simple modified Friedmann equation with the Cardassian term (Freese and Lewis 2002; Godlowski et al. 2004). Other modifications have been recently considered and tested by astronomical data, e.g. the so-called polytropic Cardassian models which can be embedded in the considered dynamical framework were considered (Li et al. 2012).

The form (6) of the dynamical system opens the possibility of adopting dynamical system methods in investigations of all possible evolutional scenarios for all possible initial conditions. Theoretical research in this area obviously shift from founding and analyzing particular cosmological solution to investigating a space of all admissible solutions and discovering how certain properties (like acceleration, existence of singularities for example) are "distributed" in this space. The system (6) is Hamiltonian one and adopting Hamiltonian formalism into the admissible motion seems to be natural. This gives at once insight into dynamics of accelerating Universe because our problem is similar to the problems of classical mechanics. It is achieved due to particle like description of accelerating cosmology. This cosmology identifies the unique form of the potential function $V(x)$. Different potential functions for different propositions of solving the acceleration problem contains Table 1.

\section{Genericity of acceleration scenario in the framework of structural stability}

The dynamical system investigation of the solutions of differential equations shifts key point from founding and analyzing of individual solutions to investigating the space of all 
Table 1 The potential function for different dark energy models

\begin{tabular}{|c|c|c|}
\hline Model & Potential function & Independent parameters \\
\hline $\begin{array}{l}\text { Einstein-de Sitter model } \\
\Omega_{\mathrm{m}, 0}=1, \Omega_{\Lambda, 0}=0 \\
\Omega_{k, 0}=0\end{array}$ & $V(x)=-\frac{1}{2} \Omega_{\mathrm{m}, 0} x^{-1}$ & $H_{0}$ \\
\hline $\begin{array}{l}\text { LCDM model } \\
\Omega_{\mathrm{m}, 0}+\Omega_{\Lambda, 0}=1\end{array}$ & $V(x)=-\frac{1}{2}\left\{\Omega_{\mathrm{m}, 0} x^{-1}+\Omega_{\Lambda, 0} x^{2}\right\}$ & $\left(\Omega_{\mathrm{m}, 0}, H_{0}\right)$ \\
\hline $\begin{array}{l}\text { FRW model filled with } \\
\text { noninteracting multifluids } \\
p=w_{i} \varrho_{i} \text { with dust, } \\
\text { matter and curvature }\end{array}$ & $\begin{aligned} V(x)= & -\frac{1}{2}\left\{\Omega_{\mathrm{m}, 0} x^{-1}+\Omega_{k, 0}\right. \\
& \left.+\sum_{i=1}^{n} \Omega_{i, 0} x^{-3\left(1+w_{i}\right)}\right\}\end{aligned}$ & $\left(\Omega_{\mathrm{m}, 0}, H_{0}, \Omega_{\Lambda, 0}, \Omega_{i, 0}\right)$ \\
\hline \multirow[t]{2}{*}{$\begin{array}{l}\text { FRW quintessence model } \\
\text { with dust and dark matter } x \\
p_{x}=w_{x} \varrho_{x}, w_{x}=\text { const }\end{array}$} & $\begin{aligned} V(x)= & -\frac{1}{2}\left\{\Omega_{\mathrm{m}, 0} x^{-1}+\Omega_{k, 0}\right. \\
& \left.+\Omega_{x, 0} x^{-1-3 w_{x}}\right\}\end{aligned}$ & $\left(\Omega_{\mathrm{m}, 0}, H_{0}, \Omega_{x, 0}\right)$ \\
\hline & $w_{x}<-1$ phantom models & $\begin{aligned} \Omega_{k, 0}= & 1-\Omega_{\mathrm{m}, 0} \\
& -\Omega_{x, 0}\end{aligned}$ \\
\hline $\begin{array}{l}\text { FRW model with baryonic } \\
\text { matter and generalized } \\
\text { Chaplygin gas }[4] \\
p=-\frac{A}{\varrho^{\alpha}}, A>0\end{array}$ & $\begin{aligned} V(x)= & -\frac{1}{2}\left\{\Omega_{\mathrm{m}, 0} x^{-1}+\Omega_{k, 0}\right. \\
& \left.+\Omega_{\mathrm{Chapl}, 0}\left(A_{s}+\frac{1-A_{s}}{x^{3(1+\alpha)}}\right) \frac{1}{1+\alpha}\right\}\end{aligned}$ & $\left(\Omega_{\mathrm{m}, 0}, H_{0}, \Omega_{\mathrm{Chapl}, 0}\right)$ \\
\hline
\end{tabular}

solutions for all admissible initial conditions, in the geometrical language of the phase space. A certain property (such as acceleration, singularities etc.) is believed to be realistic if it can be attributed to a large subset of models within the space of all solutions (Szydlowski et al. 1984). The evolutional scenarios are represented by the phase curves or by critical points, limit circles or the other limit sets. We say that two dynamical systems (or equivalently vector fields), say $f(x)$ and $g(x))$ if there is an orientation preserving a homeomorphism sending integral curves of $f$ into those of $g$. Of course this equivalence relation divides the space of all dynamical systems on the plane on disjoint class of abstraction. Let phase space $E=\mathbf{R}^{n}$, then $\epsilon$-perturbation $f$ is the function $g \in C^{1}(\mathcal{M})$ satisfying $\|f-g\|_{1}<\epsilon$; where $\mathcal{M}$ is an open subset of $R^{n}$ and $\|\ldots\|_{1}$ is a $C^{1}$ norm for the Banach space. In the introduced language it is natural to formulate an idea of structural stability. The intuition is very simple, namely $f$ is structurally stable vector field if for any vector field $f$ and $g$ are topologically equivalent. Then one can define the property of structural stability of the system.

Definition 2 A vector field $f \in C^{1}(\mathcal{M})$ is called to be structurally stable if there is an $\epsilon>0$ such that for all $g \in C^{1}(\mathcal{M})$ with $\|f-g\|_{1}<\epsilon, f$ and $g$ are topologically equivalent on open subset $R^{n}$ called $\mathcal{M}$.

The 2-dimensional case is distinguished by the fact that the Peixoto theorem (1962) gave a complete characterization of structurally stable systems on any compact, two dimensional space asserts that they are generic, i.e. forms open and dense subsets in the space of all dynamical system on the plane (Peixoto 1962).
While there are no counterpart to the Peixoto theorem in higher dimension it can be easy used to test whether such dynamical systems or cosmological origin has a structurally stable global phase portrait. In particular, a vector field on the Poincaré sphere will be structurally unstable if there are non-hyperbolic critical points at infinity on the equator of the Poincaré sphere. In the opposite case if additionally the number of critical points and limit cycles is finite $f$ is structurally stable on $S^{2}$.

In this section we will prove that the CDM model is structurally unstable (therefore exceptional in the space of all dynamical systems on the plane) and its transition (which we called emergence) to the LCDM model means perturbation of the CDM system such that new perturbed system is structurally stable (therefore generic). Moreover the LCDM system can be treated as abstract of equivalence principle (therefore representative case) introduced in the class of accelerating cosmological models. In other words all global phase portrait equivalent to LCDM cosmological models or the potential diffeomorphic to the inverted single well potential. We assume that class of the FRW dynamical systems with the dark energy can be described in terms of the single potential function of the scale factor or redshift. If dark energy is described in terms of the coefficient of the equation of state $w_{X}(z)=\frac{p_{X}}{\varrho_{X}}$ then the above assumption is always satisfied.

Let us rewrite the acceleration equation (1) in the new variable $z: 1+z=a^{-1}$. Then we obtain

$\ddot{z}=2(1+z)^{-1} \dot{z}^{2}+\frac{1}{6} \varrho(1+3 w(z))(1+z)$. 
Equation (7) represents a special case of a more general type of the equations

$\ddot{z}=f(z) \dot{z}^{2}+g(z)$.

For such a type of equations one can always eliminate the term $f(z) \dot{z}^{2}$ by the reparameterization of the original time variable $t, \cdot \equiv \frac{d}{d t}$, namely

$t \longmapsto \tau: \frac{d t}{d \tau}=h(z),{ }^{\prime} \equiv \frac{d}{d \tau}$.

For this aim it is sufficient to choose

$h(z)=\exp \left(-\int^{z} f(z) d z\right)$.

Then we rewrite (7) to the new form

$z^{\prime \prime}=g(z) \exp \left(-2 \int^{z} f(z) d z\right) \equiv-\frac{\partial V}{\partial z}$.

Equation (11) represents the evolution of the FRW dynamical system with dark energy. The potential function is given by the formula

$V(z)=-\int^{z} e^{-2 \int^{z} f(z) d z} g(z) d z$.

In the case considered $f(z)$ and $g(z)$ are determined by (7) and the potential function reduces to the form

$V(z)=-\frac{1}{6} \int \frac{\varrho(1+3 w(z))}{(1+z)^{3}} d z$,

where $\varrho$ plays the role of the effective energy density.

It is easily to check that the above formula can be exactly integrated by part if we assume that $\varrho$ satisfies the adiabatic condition (2). The final result is

$V(z)=-\frac{1}{6} \frac{\varrho}{(1+z)^{2}}$

and $z^{\prime \prime}=-\frac{\partial V}{\partial z}$, i.e. dynamics of the FRW model with dark energy reduces to the 2-dimensional dynamical system of a Newtonian type. The above equation has very simple interpretation as the motion of a particle of the unit mass in the potential well. During the motion of the system the total energy is preserved, i.e.

$\frac{\left(z^{\prime}\right)^{2}}{2}+V(z)=E=\mathrm{const}$,

where $E=-\frac{k}{2}$.

The Lagrangian of the fictitious particle which mimics the evolution of the cosmological model has the form

$\mathcal{L}=\frac{1}{2}\left(z^{\prime}\right)^{2}-V(z)$, where $\frac{d t}{d \tau} \equiv h(z)=(1+z)^{-4}$ or

$\mathcal{L}=\frac{1}{2 M^{-1}(z)}\left(\frac{d z}{d t}\right)^{2}-V(z)$,

where $M(z)=(1+z)^{4}$.

The form of the Lagrangian is natural, therefore the Hamiltonian assumes the following form

$\mathcal{H}=\frac{1}{2 M(z)} p_{z}^{2}+\tilde{V}(z)$,

where $p_{z}$ is momentum conjugated with the positional variable $z,\left(R^{\prime}\right)^{2}=-\frac{1}{2} \Omega_{k, 0}-\tilde{V}(z) \geq 0$ is a domain admissible for the motion. It is convenient to rewrite it using dimensionless variables of the density parameters instead of the energy density $\varrho_{i}$.

For this aim it is sufficient to reparameterize the time variable

$t \longmapsto\left|H_{0}\right| \tau=\sigma$,

where $H_{0}$ is the present value of the Hubble function.

Finally we obtain the Hamiltonian formulation of the dynamics of the FRW model with dark energy.

$$
\begin{aligned}
H \longmapsto \tilde{H} & =\frac{1}{2 M}\left(\frac{d z}{\left|H_{0}\right| d t}\right)^{2}+\tilde{V}(z) \\
& =\frac{1}{2 M}\left(\frac{d z}{d \sigma}\right)^{2}+\tilde{V}(z),
\end{aligned}
$$

where

$\tilde{V}(z)=-\frac{1}{2} \Omega_{\mathrm{eff}}(1+z)^{-2}, \quad E=\frac{1}{2} \Omega_{k, 0}$.

In the special cases of the CDM and LCDM models the potential function is in the form

$\tilde{V}(z)=-\frac{1}{2} \Omega_{\mathrm{m}, 0}(1+z)$

for the CDM model where $\Omega_{\mathrm{m}, 0}=1$ if $\Omega_{k, 0}=0$ (the flat model), and

$\tilde{V}(z)=-\frac{1}{2}\left[\Omega_{\mathrm{m}, 0}(1+z)+\Omega_{\Lambda, 0}(1+z)^{-2}\right]$,

for the LCDM model.

All density parameters are not independent and satisfy constraints conditions as a consequence of the conservation energy integral $\tilde{H}=E=-\Omega_{k, 0}$

$\sum_{i} \Omega_{i, 0}+\Omega_{k, 0}=1$,

where we apply $z=0$ or that

$\tilde{V}(z=0)+\frac{1}{2}=\Omega_{k, 0}$. 


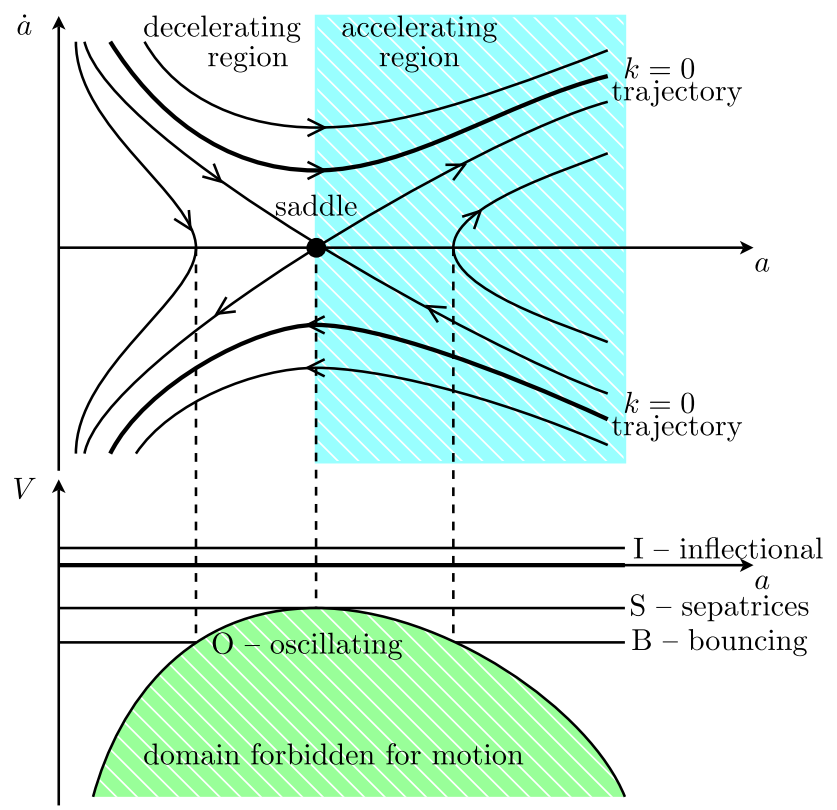

Fig. 2 The phase portrait for the perturbed potential $V(x)$ on the phase plane $\left(x, x^{\prime}\right)$ at the finite domain. Here redshift $z=x^{-1}-1$

The potential (21) can be treated as a perturbation of the potential (20) which is manifested for redshift $z<z_{\text {trans }}$. The phase portrait for the LCDM model as well as its potential $V(x)$ illustrates Fig. 2. The saddle point in the phase portrait corresponds to a maximum of the potential function. The universe is decelerating for $x<x\left(z_{\text {trans }}\right)$ and accelerating in the domain $x>x$ ( $\left.z_{\text {trans }}\right)$.

Let us define some class of the perturbed CDM models. Such a class can be defined in terms of the perturbed system of a Newtonian form. Let us stress that the notion of structural stability does not depends on the perturbation. This property is inherent property of the system itself, not perturbation.

Definition 3 By the perturbed CDM model we understand 2-dimensional dynamical system of a Newtonian form $\ddot{a}=$ $-\frac{\partial V}{\partial a}\left(\right.$ or $z^{\prime \prime}=-\frac{\partial V}{\partial z}$ ) with the potential function

$V=V_{\mathrm{CDM}}-\frac{1}{6} \sum_{0, \pm 1, \pm 2} \varrho_{i, 0} a^{-i+2}=V_{\mathrm{CDM}}+V_{\mathrm{pert}}$,

where $V_{\mathrm{CDM}}=-\frac{1}{2} \Omega_{\mathrm{m}, 0} x^{-1}, \Omega_{\mathrm{m}, 0}=1,1+z=x^{-1}$.

In (23) we define a class of perturbed LCDM systems which can be regarded as close of the LCDM model in the sense of $C^{1}$ Banach metric $\left(\varrho_{0, i}\right.$ is assumed to be small).

Table 2 contains physical interpretation of perturbation which corresponds to additional fluid contents. For different perturbation types considered only the first one (the cosmological constant) gives rise to the structural stable system.
Table 2 Different perturbations of the CDM model

\begin{tabular}{ll}
\hline$i$ & Interpretation \\
\hline 0 & cosmological constant \\
1 & 2 D topological defects $p=-\frac{2}{3} \varrho$ \\
-1 & phantoms $p=-\frac{4}{3} \varrho$ \\
-2 & superphantoms $p=-\frac{5}{3} \varrho$ \\
2 & 1D topological defects $p=-\frac{1}{3} \varrho$ \\
\hline
\end{tabular}

It is the interesting question whether the global dynamics of the CDM model is structurally stable under a perturbation term. The global structure of dynamics (phase portraits) depends on the geometry of potential function because its localization as well as character depends on the first and the second derivatives of potential function $\left(\frac{\partial V}{\partial x}\right)_{x=x_{0}}=0$, $\lambda^{2}+\left(\frac{\partial^{2} V}{\partial x^{2}}\right)_{x_{0}}=0$ respectively, where $\lambda_{1,2}$ are eigenvalues of the linearization matrix of the system and are a solution of the characteristic equation $\lambda^{2}-\operatorname{tr} A \lambda+\operatorname{det} A=0$.

We reduce the dynamics to the 2 -dimensional system in the form

$\dot{x}=y$,

$\dot{y}=-\frac{\partial V}{\partial x}$

(or $z^{\prime}=y ; y^{\prime}=-\frac{\partial V}{\partial z}$ ), where $\frac{y^{2}}{2}+V(x)=E=-\frac{1}{2} \Omega_{k, 0}=$ const is the constant of energy.

From the above equation one can be seen that all critical points (right-hand sides of the system are vanishing) are situated on the axis $x(y=0)$. From the characteristic equation we obtain that for the dynamical system under consideration only three types of critical points are admissible

1. saddle if $x_{0}:\left(\frac{\partial V}{\partial x}\right)_{x_{0}}=0$ and $\left(\frac{\partial^{2} V}{\partial x^{2}}\right)_{x=x_{0}}<0$;

2. focus if $\left(\frac{\partial^{2} V}{\partial x^{2}}\right)_{x=x_{0}}>0$;

3. degenerated critical point if $\left(\frac{\partial^{2} V}{\partial x^{2}}\right)_{x=x_{0}}=0$.

Therefore in the first case the eigenvalues are real of opposite signs, and in the second one they are purely imaginary. Because the center and degenerated (non-hyperbolic) critical points are structurally unstable only in the presence of single saddle point to guarantee the structural stability of the system at finite domain. The critical points $x_{0}$ of the perturbed system satisfy the condition

$\Omega_{\mathrm{m}, 0}=\sum_{0, \pm 1, \pm 2} \Omega_{i, 0}(2-i) x_{0}^{3-i}$.

Therefore at least there is only present such a single critical point.

Because the second derivative of the potential function is always upper convex, i.e., $\left(\frac{\partial^{2} \tilde{V}}{\partial a^{2}}\right)<0$ and critical point if 


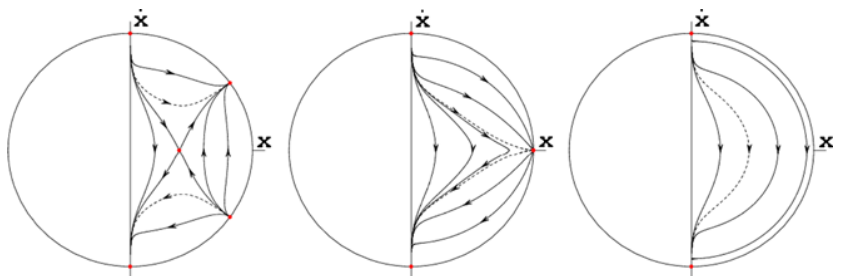

Fig. 3 The phase portraits for different perturbations of the CDM model-from the left side: (1) the LCDM model with the positive cosmological constant, (2) the LCDM model with the negative cosmological constant, (3) the CDM model with the vanishing cosmological constant. Note that only the systems with the cosmological constant are structurally stable while the CDM model is unstable because of the presence of degenerated critical points at the circle at infinity (case 3 ). The right figure represents the Einstein static universe $(x, \dot{x})=(\infty, 0)$. The critical point $(0, \infty)$ represents the big-bang singularity (an unstable node)

exists is saddle type. If we consider only Lambda term in the perturbation (i.e. the LCDM model) then other terms do not change the global phase portraits of the LCDM system or all perturbed systems are topologically equivalent. The relation of global dynamics on the phase plane is the equivalence relation, therefore the LCDM model can be treated as a representative model in this class.

After the introducing the projective map covering a circle at infinity $(x, y) \mapsto(v, w): v=\frac{1}{y}, w=\frac{x}{y}$ one can check that the system admits the critical point $\left(v_{0}=0, w_{0}=0\right)$ which corresponds to $\dot{x}=\infty$ and $\frac{1}{H}=0$ (or $H=-\infty$ ), i.e., the big-rip singularity. This critical point is degenerate, therefore the whole system is structurally unstable. The phase portraits of the CDM model and the LCDM models with an adjoint circle at infinity $x^{2}+\dot{x}^{2}=\infty$ are shown in Figs. 3,4 and 5 . $^{1}$

Note that if we consider oscillatory universes with evolution described by the center type of critical points (not the limit cycle), then such models are untypical from the point of view of the structural stability. On the other hand, if we consider the CDM models perturbed by Chaplygin gas then we obtain the phase portrait equivalent to LCDM model.

While CDM system is structurally unstable because of the presence of non-hyperbolic critical points, the LCDM model is structurally stable. The following statement will characterize structurally stable dynamical system of Newtonian type describing perturbation of CDM models.

Let us consider a more complicated $C^{\infty}$ class potential function $\tilde{V}(x)$ than a single inverted well potential (see Fig. 6) for example with two maximum points. Thus must exist minimum critical points. But its presence means that we have a center in the phase space, i.e. the system is structurally unstable.

\footnotetext{
${ }^{1}$ For comparison see Belinsky and Khalatnikov (1983, p. 32) where the corresponding phase portraits in the variables $(H, \varrho)$ were reproduced with and without the circle at infinity.
}

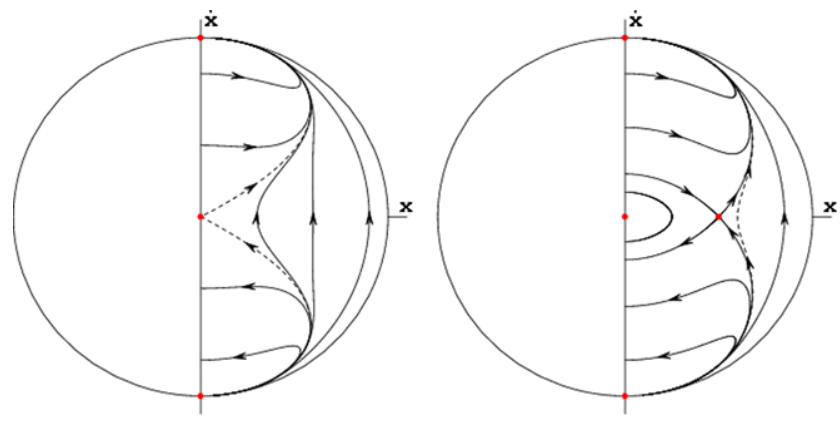

Fig. 4 The phase portraits for phantom-like perturbation of the CDM model. The both systems are structurally unstable because of the presence of degenerated critical points at infinity. The critical point $(0, \infty)$ represents a big-rip singularity characteristic for the phantom cosmology. In this case the scale factor $x$ as well as its time derivative are infinite at finite time. In the right figure appears an additional critical point of saddle type in the case of the negative cosmological constant

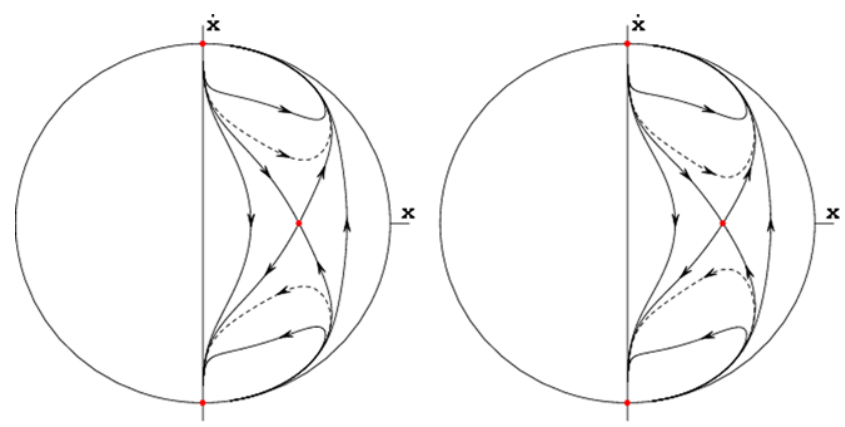

Fig. 5 The phase portraits for the both LCDM models with positive (left) and negative (right) cosmological constants perturbed by the phantom contribution. They are structurally unstable because of presence of degenerate critical points at the circle at infinity. At the critical point $(0, \infty)$ the big bang singularity is glued with the big ripe one. Note that both phase portraits are topologically equivalent

Corollary 1 If $V$ is $C^{\infty}$ function of scale factor (or redshift), then there is only one differential type of the critical point (modulo diffeomorphism) which determines the structurally stable global phase portrait.

This global dynamics is equivalent to the LCDM one. Finally the LCDM model is the simplest structurally stable generic perturbation of the CDM model which is nongeneric. The emergence of the LCDM model one can understand as a transition from a zero measure set of a dynamical system on the plane toward such which forms the open and the dense subsets in an ensemble of the dynamical systems on the plane-models of the deterministic processes.

\section{Conclusion}

The main aim of this paper was to show the effectiveness of using the framework of dynamical system theories (especially the notion of structural stability) in study of gener- 


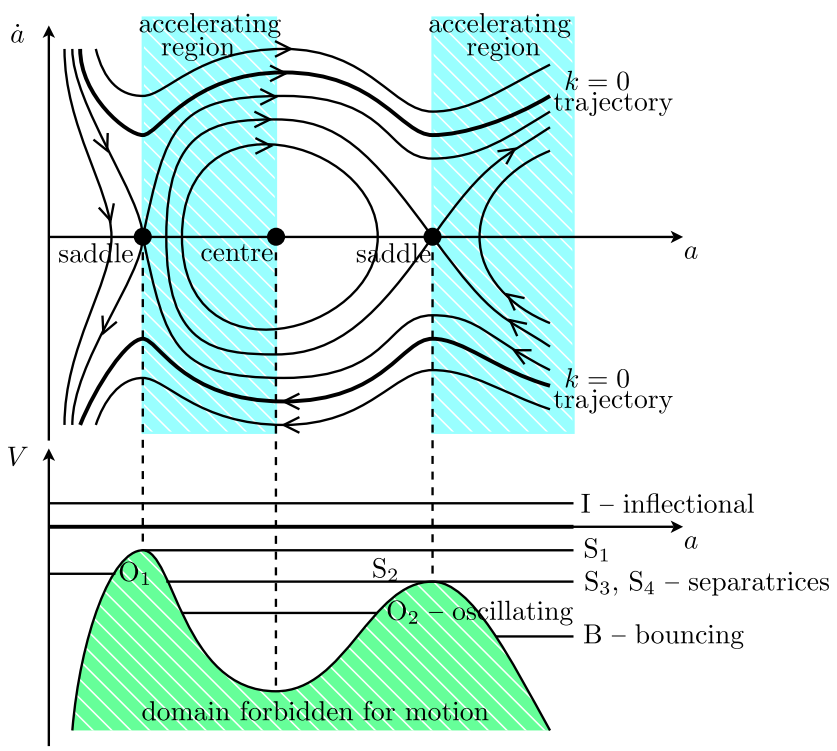

Fig. 6 More complicated evolutional scenarios on $\left(x, x^{\prime}\right)$ plane

icity of accelerated evolution of the Universe. The main result is that all transient cosmic acceleration scenarios are not generic and there is only one scenario which is dynamically equivalent to the LCDM model which is structurally stable, i.e. generic.

We always, in the mathematical modeling of physical processes, try to convey the features of typical, gardenvariety, dynamical systems. In mathematics the exceptional cases are more complicated and numerous, and they interrupt the physical discussion. Moreover dynamicists shared an opinion that such exceptional systems not arise very often because they are not typical. In the history of mathematical dynamics we observe how we have searched for generic properties. We would like to distinguish a class of phase portraits that are far simpler than the arbitrary ones. This program was achieved for dynamical systems on the plane by Peixoto due to the conception of structural stability introduced by Andronov and Leontovich in 1934. The criteria for structural stability rely upon two supplementary notions: a perturbation of the phase portraits (or vector field) and the topological equivalence (homeomorphism of the state phase). A phase portrait has the property of structural stability if all sufficiently small perturbations of it have equivalent phase portraits. For example if we consider a center type of critical points then the addition of perturbation pointing outward results in a point repeller which is not topologically equivalent to the center. This is a primary example of structurally unstable system. In the opposite case saddle type of critical point is structurally stable and the phase portrait does not change under small perturbation.

In this paper we define the class of FRW cosmological models filled by dark energy as a two-dimensional dynamical systems of a Newtonian type. They are characterized through the single smooth effective potential function of the scale factor or redshift. Among these class of models we distinguish typical (generic) and exceptional (non-generic) cases with the help of structural stability notion and the Peixoto theorem. We find that the LCDM model in opposition to the CDM model is structurally stable. We demonstrate that this model represents a typical structurally stable perturbation of CDM one. Therefore, the transition from the CDM model of the Universe toward the LCDM one, which includes the effects of the cosmological constant, can be understood as an emergence of the model from the exceptional case to the generic one. This case represents a generic model in this sense that small changes of its right-hand sides do not change the global phase portraits. In the terms of the potential, the second order differential equation one can classify different models of cosmic acceleration. It is shown that models with the accelerating phase (which follows the deceleration) are natural and typical from the point of view of the dynamical systems theory combined with the notion of structural stability.

Recently in many cosmological models dedicated to describe the very early Universe in the Planckian regime of energies (like brane models, loop quantum gravity models) it has appeared a new type of evolutional scenarios for the universe evolution with a bounce instead of an initial singularity or the acceleration phase as a transitional effect. In any case if the center type of a critical point is present in the phase space then the corresponding evolutional scenarios become structurally unstable, i.e. non-generic. We proved that if the theoretical models predict some transitional character of current acceleration then the corresponding evolutional scenario treated globally will be always non-generic. Following the Peixoto theorem non-generic solutions can be interpreted that the corresponding models in the form of differential equation form a set of zero measure in the space of all models. Therefore they are very special or nontypical. In other words models offering by new physics are fragile while the LCDM model dedicated to describe the post-Planckian evolution becomes of basic dynamical system governing the evolution.

There are many different theoretical possibilities of explaining accelerating universe in terms of dark energy (substantial approach) or using modification of gravity (non-substantial approach) (Buchert 2008; Wiltshire 2009; Copeland et al. 2006). Among all candidates the LCDM model is favored by Bayesian selection methods (Kurek and Szydlowski 2008; Lahav and Liddle 2010; March et al. 2011). These methods indicate the best model in respect to admissible data. One can ask why the LCDM model is the best one. Our answer is that the LCDM model possesses a property of simplicity and in the same time flexibility with respect to the data. The latter can be interpreted in the tools of the structural stability notion. 
The observations indicates that we live in expanding Universe with current accelerations. It seems that this acceleration phase proceeded the deceleration phase. Provided that we assume that there was no other qualitative dynamical changes in whole evolution of the universe (at early as well as late time) the LCDM model is sufficiently complex to explain such a simple evolution of the Universe. No simpler neither the more complex model can be better description of the Universe dynamics. The future evolution of our universe is eternal expansion with the accelerating phase according to the LCDM scenario. Other possible futures given by other models are unjustified because of the structural instability. Such futures are highly improbable because they require a very special fine-tuned model to the reality.

It seems that there is possibility of an ideal description of the physical reality in such a way that our model is no more a model but described reality itself. In this case the structural stability or instability does not matter. But when as in cosmology we have a bunch of models which very roughly describe the universe evolution (which are described in terms of an effective theory rather then a fundamental one) they should accommodate the reality inside the error margin generated by the perturbation. But this feature is possessed by the structural stable models only. This is an argument in favor of dealing with structural stable models in cosmology. We have found the only structural stable two-phase model of universe dynamics with a deceleration and then acceleration phase is the LCDM model.

Open Access This article is distributed under the terms of the Creative Commons Attribution License which permits any use, distribution, and reproduction in any medium, provided the original author(s) and the source are credited.

\section{References}

Aguirregabiria, J.M., Lazkoz, R.: Mod. Phys. Lett. A 19, 927 (2004). arXiv:gr-qc/0402060

Alcaniz, J.S.: Transient cosmic acceleration (2009). arXiv:0911.1087

Andronov, A.A., Pontryagin, L.S.: Dokl. Akad. Nauk SSSR 14, 247 (1937)

Astier, P., et al.: Astron. Astrophys. 447, 31 (2006). arXiv:astro-ph/ 0510447

Belinsky, V.A., Khalatnikov, I.M.: In: Benenti, S., Ferraris, M., Francaviglia, M. (eds.) Proc. of Journées Relativistes, p. 5. Pitagora Editrice Bologna, Torino (1983)

Biesiada, M.: Astrophys. Space Sci. 283, 511 (2003)

Buchert, T.: Gen. Relativ. Gravit. 40, 467 (2008). arXiv:0707.2153

Carvalho, F.C., Alcaniz, J.S., Lima, J.A.S., Silva, R.: Phys. Rev. Lett. 97, 081301 (2006). arXiv:astro-ph/0608439

Copeland, E.J., Sami, M., Tsujikawa, S.: Int. J. Mod. Phys. D 15, 1753 (2006). arXiv:hep-th/0603057

Deffayet, C., Dvali, G.R., Gabadadze, G.: Phys. Rev. D 65, 044023 (2002). arXiv:astro-ph/0105068

Ellis, G.F.R.: In: Bertotti, B., de Felice, F., Pascolini, A. (eds.) Proc. of the GR10 Conference, p. 50. Configlio Nazionale delle Richerche, Roma (1983)

Freese, K., Lewis, M.: Phys. Lett. B 540, 1 (2002). arXiv:astro-ph/ 0201229
Frieman, J.A., Hill, C.T., Stebbins, A., Waga, I.: Phys. Rev. Lett. 75, 2077 (1995). arXiv:astro-ph/9505060

Gerstenhaber, M.: Ann. Math. 79, 59 (1964)

Godlowski, W., Szydlowski, M., Krawiec, A.: Astrophys. J. 605, 599 (2004). arXiv:astro-ph/0309569

Golda, Z.A., Szydlowski, M., Heller, M.: Gen. Relativ. Gravit. 19, 707 (1987).

Holton, G.: In: Heath, A.F. (ed.) Scientific Explanation. Clarendon Press, Oxford (1981)

Kokarev, S.S.: Gen. Relativ. Gravit. 41, 1777 (2009). arXiv:0810.5080

Kurek, A., Szydlowski, M.: Astrophys. J. 675, 1 (2008). arXiv:astro$\mathrm{ph} / 0702484$

Lahav, O., Liddle, A.R.: The Cosmological Parameters 2010 (2010). arXiv: 1002.3488

Li, Z., Wu, P., Yu, H.: Astrophys. J. 744, 176 (2012). arXiv:1109.6125

Lidsey, J.E.: Gen. Relativ. Gravit. 25, 399 (1993).

Losee, J.: A Historical Introduction to the Philosophy of Science. Oxford University Press, Oxford (1993)

March, M.C., Trotta, R., Berkes, P., Starkman, G.D., Vaudrevange, P.M.: Improved constraints on cosmological parameters from SNIa data (2011). arXiv:1102.3237

Padmanabhan, T.: Structure Formation in the Universe. Cambridge University Press, Oxford (1993)

Peebles, P.J.E.: Principles of Physical Cosmology. Princeton University Press, Princeton (1993)

Peixoto, M.M.: Topology 1, 101 (1962)

Perlmutter, S., et al.: Nature 391, 51 (1998). arXiv:astro-ph/ 9712212

Perlmutter, S., et al.: Astrophys. J. 517, 565 (1999). arXiv:astro-ph/ 9812133

Riess, A.G., et al.: Astron. J. 116, 1009 (1998). arXiv:astro-ph/ 9805201

Riess, A.G., et al.: Astrophys. J. 607, 665 (2004). arXiv:astro-ph/ 0402512

Riess, A.G., Press, W.H., Kirshner, R.P.: Astrophys. J. 438, 17 (1995). arXiv:astro-ph/9410054

Roger, C.: Gaz. Math. 49, 75 (1991)

Sahni, V., Shtanov, Y.: J. Cosmol. Astropart. Phys. 0311, 014 (2003). arXiv:astro-ph/0202346

Shafieloo, A., Sahni, V., Starobinsky, A.A.: Phys. Rev. D 80, 101301 (2009). arXiv:0903.5141

Shtanov, Y.V.: On brane-world cosmology (2000). arXiv:hep-th/ 0005193

Shtanov, Y., Sahni, V.: Phys. Lett. B 557, 1 (2003). arXiv:gr-qc/ 0208047

Spergel, D.N., et al.: Astrophys. J. Suppl. Ser. 148, 175 (2003). arXiv:astro-ph/0302209

Szydlowski, M.: J. Cosmol. Astropart. Phys. 0709, 007 (2007). arXiv:astro-ph/0610250

Szydlowski, M., Czaja, W.: Phys. Rev. D 69, 083518 (2004a). arXiv:gr-qc/0305033

Szydlowski, M., Czaja, W.: Phys. Rev. D 69, 023506 (2004b). arXiv:astro-ph/0306579

Szydlowski, M., Czaja, W.: Phys. Rev. D 69, 083507 (2004c). arXiv:astro-ph/0309191

Szydlowski, M., Heller, M., Golda, Z.: Gen. Relativ. Gravit. 16, 877 (1984).

Tavakol, R.K., Ellis, G.F.R.: Phys. Lett. A 130, 217 (1988)

Tegmark, M., et al.: Phys. Rev. D 69, 103501 (2004). arXiv:astro-ph/ 0310723

Thom, R.: Stabilité Structurelle et Morphogénèse. InterÉditions, Paris (1977)

Wiltshire, D.L.: Gravitational energy as dark energy: average observational quantities (2009). arXiv:0912.5236 